\title{
Binge Drinking and Unplanned Sexual Behaviour: Deconstructing the Role of Impulsivity
}

\author{
Jane E. Scott, Julia M. Townshend, and Frances J. Hunt
}

School of Human and Social Sciences, University of West London, Paragon House, Boston Manor Road, Brentford, Middlesex, TW8 9GA, UK

Address correspondence to Jane E.Scott, jane.scott@uwl.ac.uk

Received 08 November 2018; Revised 13 January 2019; Accepted 14 January 2019

Copyright (C) 2018 Jane E. Scott, et al. This is an open access article distributed under the terms of the Creative Commons Attribution License, which permits unrestricted use, distribution, and reproduction in any medium, provided the original work is properly cited.

\begin{abstract}
The co-occurrence of binge drinking and unplanned sexual behaviour (USB) is a physiological and social concern; however, potential underlying mechanisms in this relationship remain largely unexplored. The current study compared low and high-binge drinkers on impulsivity variants and USB. Participants were 122 university students (71 females). Questionnaires measured binge drinking, USB, reward sensitivity, and trait impulsivity (Barratt Impulsiveness Scales). Cognitive-behavioural aspects of impulsivity were assessed using a Stop Signal Task (response inhibition) and an Information Sampling Task (IST: reflection-impulsivity). ANOVAs revealed that high-binge drinkers scored more impulsively than low-bingers on self-report impulsivity, and the decreasing win condition of the IST. A positive relationship was found between USB and self-report, but not cognitive-behavioural, impulsivity. In regression analysis, both binge drinking and trait impulsivity were found to have a unique effect on the proclivity to engage in USB. Findings provide an insight into demarcating impulsivity's relationship with both binge drinking and USB.
\end{abstract}

Keywords: binge drinking; unplanned sexual behaviour; trait impulsivity; reflection-impulsivity; response inhibition; reward sensitivity

\section{Introduction}

Repeated episodes of binge-style drinking (approximately four or more drinks for females and five or more drinks for males within a two-hour period [1]) has been posited to be particularly deleterious to neurocognitive functioning and puts the individual at an increased vulnerability for long-term health problems and addiction [2]. Often associated with young adult students $[3,4]$, this pattern of episodic alcohol consumption has also been implicated in an array of adverse consequences including poor academic performance, criminal involvement, drink-driving, perpetrating or being victims of physical or sexual assault, and unplanned and unsafe sexual activity [5].
Unplanned sexual behaviour (USB) refers to the act of engaging in a brief sexual encounter with someone outside of a committed relationship. Sometimes referred to as casual sex or a one-night stand, this category of sexual activity typically occurs with someone only once and is usually devoid of commitment or emotional involvement [6]. Concernedly, some studies have found that when binge drinking precedes unplanned sex, both males and females are more inclined to participate in unprotected sexual activity, thereby increasing their risk of adverse outcomes such as unplanned pregnancy, sexually transmitted infections (STIs), and the Human Immunodeficiency Virus (HIV) [7].

Previous research attempting to explore the underlying mechanisms in the relationship between binge drinking and USB has focused on various theories including alcohol myopia [8] and alcohol expectancies [9]. In addition, potential risk factors such as family background, peer influence, and personality traits, including sensation seeking and impulsivity, have all been considered [10]. Of interest here is the multi-dimensional construct of impulsivity, which has previously been associated with both USB and binge drinking respectively $[11,12]$.

Regarding sexual behaviour, both unplanned and unprotected sexual activity have been associated with various facets of trait impulsivity including negative and positive urgency, (a lack of) premeditation, sensation seeking, and impulsive decision-making [11,15]; although it should be noted that results have varied depending on the sample and criteria used for unplanned or 'risky' sexual behaviour. In addition, studies examining neurocognitive performance on inhibitory control have evidenced a positive relationship between impulsive behaviour and sexual risk-taking $[16,17,18]$. 
In respect of binge drinking, varying dimensions of impulsivity have been implicated in both the initiation and continuation of binge drinking. For example, an impulsive personality may predispose an individual to drink to excess and from an early age and, in return, repeated intoxication and withdrawal from ethanol can affect prefrontal neural systems responsible for inhibitory control. This in turn can further exacerbate impulsive behaviour and a tendency towards binge drinking creating a reciprocal effect $[13,14]$. As such, the importance of testing for both trait and behavioural measures of impulsivity is invaluable in exploring the relationship with both binge drinking and USB.

To date, however, research integrating the three elements has been limited. Both Gullette and Lyons [19] and Donohew et al. [20] discovered a positive relationship for impulsivity variants, such as sensation seeking and impulsive decision-making, in co-occurring alcohol and risky sexual behaviour. In a similar vein, a mediating role for both sensation seeking [21] and excitement seeking [22] has been demonstrated in the relationship between alcohol use and high-risk sexual behaviour. Regarding behavioural impulsivity, a study by MacKillop et al. [22] found a relationship between impulsive responding on a delay discounting task and increased risky sexual behaviour during alcohol intoxication.

Nevertheless, a limitation of this research has been the utilization of single measures of impulsivity and not accounting for the binge-style consumption of alcohol. A previous study by Townshend, Kambouropoulos, Griffin, Hunt, and Milani [23] incorporated a cognitivebehavioural measure of impulsivity to assess levels of reflection-impulsivity (i.e., the ability to gather and evaluate information during decision making), as well as a single question to measure self-reported impulsivity, and examined the relationship between binge drinking and USB. Results revealed that the high-binge drinkers engaged in more unplanned sexual encounters, rated themselves as more impulsive, and demonstrated aspects of reflection-impulsivity compared to the low-binge drinkers, although no comprehensive trait measure of impulsivity was included in their study.

Taking into consideration the ubiquity of both binge drinking and unplanned sexual activity in university students during emerging adulthood [24], a clearer understanding of potential mediating factors, such as impulsivity, would be advantageous in efforts intended to reduce unwanted pregnancy, STIs, and coerced sexual activity, as well as alcohol-related problems and dependence. Thus, the overriding aim of the current study was to explore the relationship between binge drinking, USB, and impulsivity using an extensive battery of behavioural and self-report measures to examine the various dimensions of impulsivity. Accordingly, a sample of low and high-binge drinkers, derived from the Alcohol Use Questionnaire [25], were compared on different dimensions of impulsivity, as well as the tendency to engage in USB.

Specifically, based on previous findings in the literature, it is predicted that the high-binge drinking group will score higher on self-report measures of impulsivity, display poorer response inhibition, and show signs of less reflection and more impulsiveness compared to the low-binge drinking group. In addition, it is predicted that the high-binge drinking group will report more episodes of USB, compared to the lowbinge drinking group. Furthermore, it is predicted that more impulsive individuals will report more episodes of USB, compared to less impulsive individuals. Finally, based on previous research [23], an interaction will be explored between binge drinking and impulsivity, and the tendency to engage in USB.

\section{Methods}

\subsection{Participants}

Participants $(\mathrm{N}=125)$ were recruited through inclass announcements and university website. Criteria included being aged between 18 and 30 and describing oneself as a "social drinker" (occasional through to heavy consumption) but excluded those with a history of drug or alcohol dependence. Participants, all full-time Psychology students, provided relevant demographic details, including age of first drink and age of first intoxication. Participants consuming more than eight units of alcohol in the preceding 24 hours were excluded from the study. This last criterion excluded three participants leaving a total of 122 participants $\left(\mathrm{M}_{\text {age }}=\right.$ $21.30, \mathrm{SD}_{\text {age }}=3.54 ; 58 \%$ female).

\subsection{Measures}

All questionnaires used in the study are robust measures that have been employed in numerous studies and are recognized as having high levels of reliability and validity. The one exception is the Unplanned Sexual Behaviour Questionnaire (USBQ) that was utilized in the previous study [see 23].

\subsubsection{Alcohol Use Questionnaire (AUQ [25]):}

A revised version of the AUQ [26] was used to establish drinking behaviour. The AUQ provides a binge score, as well as total alcohol units consumed per week. The binge score is calculated from the number of times being drunk (previous six months), percentage of times getting drunk when drinking (average), and speed of drinking (average drinks per hour). The median was then calculated from the binge score (22.5) and used to form two groups above and below the median: high-binge drinkers $(\geq 23 ; n$ $=61)$ and low-binge drinkers $(\leq 22 ; n=61)$ respectively.

\subsubsection{Unplanned Sexual Behaviour Questionnaire (USBQ [23]):}

The 16-item USBQ asks questions relating to unplanned sexual behaviour, decision making, 
impulsivity, and regret. The two questions of relevance to this paper included: Q1, “Approximately how many times have you ever engaged in unplanned sexual activity with non-partners or strangers?". Possible answers are on a scale from "never", "once", "2-5 occasions", "610 occasions", to "11 or more occasions". Secondly, Q13 asks: "Generally, would you describe yourself as an impulsive person?". Answers are on a 5-point Likert scale ( $1=$ "not at all impulsive" to $5=$ "very impulsive").

2.2.3 Barratt Impulsiveness Scale, Version 11 (BIS-11 [27]):

The 30-item BIS-11 assesses the personality (trait) dimensions of impulsivity. For example, "I do things without thinking". Answers are on a 4-point Likert scale ranging from "rarely/never" to "almost always/ always". Several items are reverse scored and the greater the summed score, the higher the self-reported level of impulsivity. In addition to the total score, the BIS-11 provides three subscales including attentional, motor, and non-planning facets of impulsivity. Cronbach's alphas for the current sample were .69 (attentional), .60 (motor), 69 (non-planning) and .82 (total score).

\subsubsection{Sensitivity to Reward Questionnaire (SPSRQ-SR} [28]):

This measure of reward sensitivity requires participants to tick "yes" or "no" to 17 items. For example, "Do you often do things to be praised?". A "yes" response is assigned a value of one and a "no" response a zero, and then summed to form a SR scale score. The greater the summed score, the higher the selfreported level of reward sensitivity. The Cronbach's reliability coefficient was .74 for the current sample.

2.2.5 Reflection Impulsivity: Information Sampling Task (IST; CANTAB Cambridge Cognition Ltd.):

The IST assesses reflection-impulsivity on two sets of ten trials. Twenty-five grey boxes are presented on a $5 \times 5$ matrix with two differently coloured squares displayed beneath. When respondents touch any of the grey squares they turn to one of the two colours displayed below and remain that colour for the duration of each individual trial, so there is no working memory requirement to the task. Participants are asked to decide which colour is in the majority, basing their decision on the boxes revealed.

The first set of ten trials is the Fixed Win (FW) condition with a win of 100 points for a correct choice, and a deduction of 100 points for an incorrect choice. Participants are informed that they can open as many boxes as they wish. The second trial is the Decreasing Win (DW) condition in which participants start with 250 points; however, this time the score decreases by ten points with every box opened, consequently the earlier a decision is made the more points are awarded, providing the selection is correct. As before, one hundred points is deducted for a wrong decision. Performance on the two tasks is measured by the number of boxes opened per trial, proportion of correct choices $[P($ correct $)$; the mean probability of being correct at the point of decision], number of errors committed when selecting the colour, and time taken to make a decision (opening latency).

2.2.6 Response inhibition: Stop Signal Task (SST; CANTAB Cambridge Cognition Ltd.):

The SST provides a measure of an individual's ability to inhibit a prepotent response. Participants are instructed to select the right or left-hand button on a press pad in accordance with the right or left-pointing directional arrow presented on the screen in front of them. Following this trial phase, the participant is then instructed to withhold their response if they hear an auditory signal. The 'stop signal' (beep) is randomized and occurs on $25 \%$ of trials with a variable 'stop signal delay' (SSD) between the onset of the arrow stimulus and the auditory tone. The variation of the SSD is dependent on the participant's performance, but adjusted so that 'stopping' occurs approximately $50 \%$ of the time for all participants. Performance on this task is measured by the number of directional errors (DE), median response time on GO trials (GoRT), and the stop-signal reaction time (SSRT), which is an estimate of the time between the go and stop stimuli that the participants has successfully inhibited their response $50 \%$ of the time (a higher SSRT score indicates poor inhibitory control).

\subsection{Procedure}

The study was approved by the university's ethics subcommittee. Assurances were given regarding anonymity, confidentiality and participants' right to withdraw. Participants provided informed consent and were awarded research participation pool points for their time. Participants were instructed to work through a battery of questionnaires interspersed with two computer tasks. These were presented in the order of: demographics, BIS-11, SST, SPSRQ-SR, USBQ, IST, and AUQ, with the order of the IST and SST being reversed between participants. All procedures took place in a dedicated research laboratory onsite and lasted approximately 50 minutes.

\section{Results}

\subsection{Demographics}

Table 1 shows the demographic data for the low and high-binge drinking groups, and separately for males and females. The high-binge drinkers drank more units of alcohol per week, $t(87.06)=-4.14, p<.001$, and first became drunk at a younger age, $t(116)=2.16, p=$ .033. There was also a difference between the ages of the groups with the high-binge drinkers being slightly younger compared to the low-binge drinkers, $t(85.57)=$ $3.66, p<.001$. Gender was added to check for a potential confound effect; however, there were no significant 
Table 1: Demographic and alcohol use data for low-binge and high-binge drinkers and for males and females within each group.

\begin{tabular}{|c|c|c|c|c|c|c|}
\hline \multirow{2}{*}{ Group characteristics } & \multicolumn{3}{|c|}{ Low-binge drinkers } & \multicolumn{3}{|c|}{ High-binge drinkers } \\
\hline & Total & Males & Females & Total & Males & Females \\
\hline Number & 61 & 21 & 40 & 61 & 30 & 31 \\
\hline $\mathrm{Age}^{* * *}$ & $22.41(4.31)$ & $22.05(3.63)$ & $22.60(4.66)$ & $20.18(2.04)$ & $20.57(2.34)$ & $19.81(1.64)$ \\
\hline Alcohol units ${ }^{1}$ per week ${ }^{* * *}$ & $10.03(7.21)$ & $12.20(8.58)$ & $8.89(6.19)$ & $18.73(14.77)$ & $20.05(15.98)$ & $17.45(13.63)$ \\
\hline Binge drinking score $* * *$ & $12.85(5.66)$ & $13.41(5.53)$ & $12.56(5.78)$ & $47.21(22.32)$ & $48.33(25.09)$ & $46.13(19.64)$ \\
\hline Age of first drink & $14.48(3.17)$ & $14.57(3.67)$ & $14.43(2.92)$ & $14.08(2.69)$ & $14.20(2.67)$ & $13.97(2.75)$ \\
\hline Age of first time being drunk* & $16.39(1.98)$ & $16.84(2.06)$ & $16.16(1.93)$ & $15.52(2.32)$ & $15.80(2.06)$ & $15.26(2.56)$ \\
\hline
\end{tabular}

Note. ${ }^{1}$ One unit is $8 \mathrm{~g}$ of alcohol. Data are presented as mean (SD). ${ }^{*} \mathrm{p}<0.05,{ }^{* *} \mathrm{p}<0.001$ differences between groups $($ low/high-binge drinkers).

differences between males and females on the binge drinking score, $t(120)=1.56, p=.122$, and just a trendlevel difference on alcohol units per week, $t(120)=1.87$, $p=.065$, demonstrating a slightly higher alcohol intake reported for males compared to females.

\subsection{Self-report impulsivity measures}

A one-way MANOVA examined group differences on all self-report measures of impulsivity, except for the BIS-11 total score, and demonstrated an overall main effect of binge drinking group [Wilks' Lambda $=.81$, $\left.F(5,116)=5.30, p<.001, \eta_{p}^{2}=.19\right]$. Univariate analyses revealed differences between low and high-binge drinkers, using a Bonferroni adjusted alpha of .001, on sensitivity to reward, $F(1,120)=13.15, p<.001, \eta^{2}=$ .10 , motor impulsivity, $F(1,120)=13.01, p<.001, \eta_{p}^{p}=$ .10 , and non-planning impulsivity, $F(1,120)=15.07, p<$ $.001, \eta_{p}^{2}=.11$. A trend-level difference was revealed for the "how impulsive are you" question from the USBQ, $F(1,120)=6.18, p=.014, \eta_{p}^{2}=.05$. There was no effect of binge drinking on attentional impulsivity, $F(1,120)=$ $3.79, p=.054, \eta_{p}^{2}=.03$. A separate univariate analysis was conducted between the BIS-11 total score and binge group due to the high correlation between the BIS-11 total score and the other BIS-11 subscales $(r \geq .75)$. Results revealed a significant difference between binge drinking groups, $F(1,120)=16.29, p<.001, \eta_{p}^{2}=.12$. In all selfreport impulsivity measures the high-binge drinking group scored higher than the low-binge drinking group.

3.3 Reflection-impulsivity: Information Sampling Task (IST)

The IST analysis was conducted in two parts: firstly, a trial by trial analysis involving a series of one-way ANOVAs to examine potential binge group differences on number of boxes opened, $P$ (correct), total errors and latency, and secondly, a within-subjects analysis to determine a potential effect of condition on performance across the FW and DW trials.

As Table 2 demonstrates, binge drinking groups were found to perform similarly on all IST variables in the FW condition. In contrast, the DW condition revealed a significant difference between low and highbinge drinking group for boxes opened and $P$ (correct). Examination of the means reveals that the high-binge drinkers opened fewer boxes than the low-binge drinkers, and that their probability of being correct was lower than for the low-binge drinkers.

A series of mixed-model ANOVAs were then conducted to examine potential differences across the FW and DW conditions (data not shown). There was a significant difference between the FW and DW condition on all IST variables ( $\left.p s<.001, \eta_{p}^{2}>.42\right)$, demonstrating that overall all participants altered their performance across the FW and DW conditions. This finding suggests a sensitivity to the altered reward characteristics between the two conditions and a desire to win more points. No differences were revealed between binge drinking groups on any of the IST variables ( $p s>.05, \eta_{p}{ }_{p}<.03$; combined FW and DW scores).

\subsection{Response inhibition: Stop Signal Task (SST)}

Independent t-tests were conducted to examine group differences on the SST variables [direction errors (DE), median reaction time on Go trials (GoRT), and StopSignal Reaction time (SSRT)]. Results revealed one group difference for DE $(p=.028$, Cohen's $d=.41)$ demonstrating that the high-binge group committed more directional errors than the low-binge group (e.g., pressing the left button for a right arrow). There were no binge drinking group differences for GoRT or SSRT ( $\left.p s^{>} .05\right)$.

\subsection{Unplanned sexual behaviour questionnaire (USBQ)}

A one-way ANOVA was conducted to examine group differences for times reported engaging in unplanned sexual behaviour (USB). Results revealed a significant difference for binge drinking group, $F(1,120)=4.76, p$ $=.031, \eta_{p}^{2}=.04$, with the high-binge drinkers $(M=2.70$, $S D=1.36$ ) reporting more episodes of USB than the lowbinge drinkers $(M=2.18, S D=1.30)$. In further analysis, a bivariate correlation between all self-report measures of impulsivity and the number of times engaged in USB revealed a positive association with all variables, except the BIS-11 attentional scale (see Table 3). No relationship was revealed between the number of times engaged in USB and either of the IST or SST outcomes. These findings suggest that a proclivity to engage in USB is related to higher scores for reward sensitivity, motor and non-planning impulsivity, and the self-reported impulsivity question from the USBQ (Q13), but that it is not associated with the dimensions of impulsivity elicited by behavioural and cognitive measures. 
Table 2: Mean, standard deviation and group differences between low and high-binge drinkers on the Information Sampling Task (N=122).

\begin{tabular}{|c|c|c|c|c|c|c|c|}
\hline \multirow{2}{*}{$\begin{array}{l}\text { Information Sampling Task } \\
\text { variables }\end{array}$} & \multicolumn{2}{|c|}{ Low-binge drinkers $(n=61)$} & \multicolumn{2}{|c|}{ High-binge drinkers $(n=61)$} & \multirow{2}{*}{$F(1,120)$} & \multirow[b]{2}{*}{$p$} & \multirow{2}{*}{$\eta_{p}^{2}$} \\
\hline & M & SD & M & SD & & & \\
\hline FW Boxes opened & 15.05 & 6.08 & 14.47 & 6.21 & .27 & .603 & .00 \\
\hline FW $P$ (correct) & .81 & .11 & .81 & .12 & .00 & .968 & .00 \\
\hline FW Total Error & 1.51 & 1.31 & 1.33 & 1.26 & .60 & .440 & .01 \\
\hline FW Latency $(m s)$ & 961.10 & 800.92 & 850.27 & 450.07 & .89 & .348 & .01 \\
\hline DW Boxes opened & 8.69 & 3.40 & 7.02 & 2.91 & 8.56 & .004 & .07 \\
\hline DW $P$ (correct) & .70 & .07 & .67 & .07 & 6.43 & .013 & .05 \\
\hline DW Total Error & 2.70 & 1.46 & 3.10 & 1.45 & 2.23 & .138 & .02 \\
\hline DW Latency $(m s)$ & 1272.28 & 653.54 & 1392.20 & 657.30 & 1.02 & .314 & .01 \\
\hline
\end{tabular}

Note. FW = Fixed Win condition; DW = Decreasing Win condition; = partial eta squared.

Table 3: Pearsons' correlation matrix between number of times engaged in unplanned sexual behaviour and all self-report measures of impulsivity.

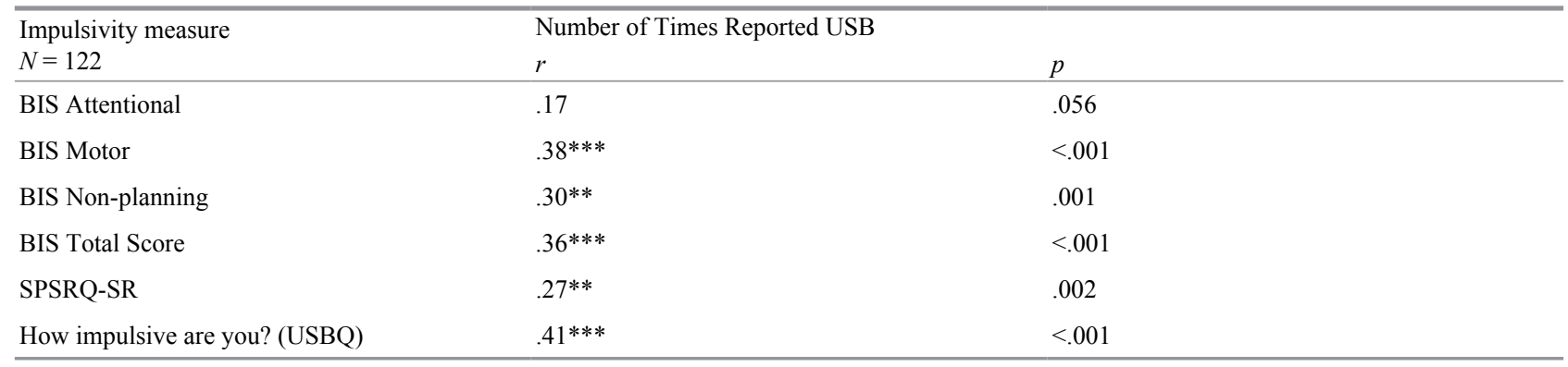

Note. ${ }^{* *} \mathrm{p}<0.01,{ }^{* * *} \mathrm{p}<0.001$. BIS = Barratt Impulsiveness Scale, version 11; SPSRQ-SR - Sensitivity to Reward Questionnaire; USBQ - Unplanned Sexual Behaviour Questionnaire.

Table 4: Hierarchical regression exploring interaction between impulsivity and binge drinking on times reported engaging in unplanned sexual behaviour.

\begin{tabular}{|c|c|c|c|c|c|c|c|c|}
\hline Step and predictor variable & $\mathrm{B}$ & $S E B$ & $\beta$ & $R^{2}$ & $\Delta R^{2}$ & $t$ & $p$ & $s r^{2}$ \\
\hline Step 1: & & & & .18 & $.17 * * *$ & & & \\
\hline Binge score ${ }^{a}$ & .02 & .01 & .26 & & & 2.83 & .006 & .05 \\
\hline BIS Total $^{\mathrm{a}}$ & .03 & .01 & .26 & & & 2.86 & .005 & .06 \\
\hline Step 2: & & & & .19 & .00 & & & \\
\hline Binge score ${ }^{a}$ & .02 & .01 & .28 & & & 2.81 & .006 & .05 \\
\hline BIS Total $^{\mathrm{a}}$ & .03 & .01 & .25 & & & 2.79 & .006 & .05 \\
\hline BIS Total $x$ Binge $^{a}$ & .00 & .00 & -.06 & & & -.60 & .553 & .00 \\
\hline
\end{tabular}

Note. $* * * \mathrm{p}<.001 .^{\mathrm{a}}=$ variables centred to avoid multicollinearity.

\subsection{Regression analysis}

A final analysis (Table 4) was conducted to examine the predictive relationship between binge drinking, impulsivity and USB. The selection of predictors was determined by the power of the aforementioned results (i.e., largest $\eta_{p}^{2}$ ). Preliminary analyses ensured no violations were committed pertaining to assumptions of normality, linearity, and homoscedasticity. It was anticipated that trait impulsivity may interact with binge drinking to influence engaging in USB, therefore a product term was constructed from the binge score and the BIS-11 total score prior to analysis. Both binge score and the BIS-11 total score were mean-centred prior to analysis and the interaction term was constructed from the mean-centred variables to minimise multicollinearity. Binge score and the BIS-11 total score were entered in Step 1 and explained $18.3 \%$ of the variance in times engaged in USB. The product term of binge score and BIS- 11 total score was entered in Step 2 and explained an additional $.35 \%$ of variance in times engaged in USB.
In the final model, both binge drinking and the BIS-11 total score were significant predictors of times engaged in USB; however, the product term of trait impulsivity and binge score was not significant (see Table 4).

\section{Discussion}

The results are generally in line with prediction and revealed that a sample of heavier binge drinkers exhibited higher levels of trait impulsivity, reward sensitivity, and demonstrated impairments on a cognitive-behavioural task designed to measure reflection-impulsivity. In addition, the high-binge drinkers reported more episodes of unplanned sexual behaviour (USB), in comparison to low-binge drinkers. Furthermore, more impulsive individuals were found to report higher levels of USB, although interestingly, no interaction was demonstrated between impulsivity and binge drinking scores on episodes of USB in a regression analysis. Indeed, the results found here suggest that both binge drinking and an impulsive personality may be uniquely related to the tendency to engage in USB. 
There is a plethora of research finding a positive relationship between alcohol use and USB and moreover, heightened impulsivity, or variants such as sensation seeking, have previously been implicated in the relationship with risky sex [10]. However, there has been a paucity of research incorporating binge drinking, USB and both trait and behavioural impulsivity in a single study, and as such, these findings add to the existing literature; although further replication of these findings is recommended. Nevertheless, the current study does corroborate with the previous one by Townshend et al. [23], which found that high-binge drinkers reported more episodes of USB and scored higher on a single self-report impulsivity question, in comparison to the low-binge drinkers.

The discovery that the high-binge drinkers displayed higher levels of trait impulsivity and reward sensitivity is congruent with previous research [29, 30]. Specifically, the high-binge drinking group scored higher on nonplanning and motor impulsiveness, suggesting a lack of forethought and the tendency to act on the spur of the moment without regard for adverse consequences. In addition, the high-binge drinkers reported higher levels on a reward sensitivity measure suggestive of a heightened appetitive motivation towards binge drinking. Indeed, these findings confirm previous factor analytic research proposing two distinct components of impulsivity (i.e., rash-spontaneous impulsivity and reward sensitivity) that appear to be positively associated with alcohol or substance use [31].

Further evidence for the multi-dimensional construct of impulsivity was demonstrated by the finding that the high-binge drinkers opened fewer boxes and thus, significantly lowered their probability of being correct $[P($ correct $)]$ in the Decreasing Win (DW) condition of the IST (where points were deducted for each subsequent box opened). These findings partially corroborate with previous studies; for example, the previous study by Townshend et al. [23] demonstrated a similar difference between high and low binge drinkers, although this was revealed in the Fixed Win (FW), not the DW condition. Research elsewhere has been mixed, with some studies demonstrating a group difference between alcohol or cannabis users and healthy controls on both conditions of the IST [32, 33], whereas others have failed to find a difference on either condition [34, 29]. Nevertheless, the results found here are congruent with a study by Bø, Aker, Billieux, and Landrø [35], who found that binge drinking was a significant predictor of the probability of being correct $[P($ correct $)]$ in the DW condition, and not the FW. This outcome suggests that the high-binge drinkers may have played the task more strategically than the low-binge drinkers, with a group difference only transpiring with the opportunity to accrue more points. As Bø et al. [35] assert, this may suggest that the binge drinkers are more sensitive to the positive consequences of the DW condition, rather than impaired decision making and evaluating information per se (the central tenets of reflection-impulsivity).

An additional behavioural task, the SST, carried out to determine a difference between binge drinking groups on response inhibition was not significant. This study failed to demonstrate a group difference on either GoRT (the median response time on Go trials), or the key measure of stop-signal reaction time (a higher SSRT is an index of impaired response inhibition). However, the finding that high-binge drinkers committed more directional errors, in comparison to low-binge drinkers, is in line with previous research demonstrating an impaired performance on go accuracy errors from heavy drinkers [36]. Further research is warranted, but these findings suggest that inaccuracy in performance on the SST may be indicative of a difference in attention or cognitive impairment, which may be exacerbated in high-binge drinkers. The lack of outcome on the SSRT variable may reflect the characteristics of this sample (i.e., young adult social drinkers) as opposed to alcohol dependents, who have previously been shown to demonstrate increased response inhibition [33].

A limitation of the current study is the cross-sectional design, thus prohibiting a causal inference from the findings. Also, worthy to note is that the current sample was taken from a single university in the UK and thus, findings may not generalise to other geographical or nonstudent populations. Inarguably, taking into consideration the cohort of young adult students, the co-occurrence of binge drinking and USB may be high anyway. However, an important contribution to the existing literature in this area was the demonstration that elevated levels of trait impulsivity were positively related to both binge drinking and times reported to engage in USB. Furthermore, the finding that either high levels of trait impulsivity or binge drinking may be predictive of USB is worthy of further attention.

Previous research has suggested that heightened impulsivity from a young age may engender an individual to engage in early experimentation of risky behaviours, such as binge drinking and unplanned and unprotected sexual activity [37]. In addition, prolonged binge drinking has been evidenced to effect neural systems responsible for inhibitory control, thus exacerbating impulsive behaviour and potentially creating a reciprocal cycle $[13,14]$. As such, early identification of individuals high in impulsivity may be advantageous in efforts and treatment programs intended to reduce risky-type behaviours. In sum, the current study's findings support the supposition that impulsivity is multi-dimensional and help to expand previous research demonstrating that specific aspects of impulsivity may be influential in the relationship with both binge drinking and USB. 


\section{Funding}

This research did not receive any specific grant from funding agencies in the public, commercial, or not-forprofit sectors.

\section{Conflicts of interest}

The authors declare that they have no conflict of interest.

\section{References}

[1]. National Institute on Alcohol Abuse and Alcoholism, Binge drinking defined, NIAAA Newsletter, 3 (2004).

[2]. P. Maurage, F. Joassin, A. Speth, J. Modave, P. Philippot, and S. Campanell, Cerebral effects of binge drinking: respective influences of global alcohol intake and consumption pattern, Clin Neurophysiol, 123 (2012), 892-901.

[3]. A. C. Carter, K. O. Brandon, and M. S. Goldman, The college and noncollege experience: a review of the factors that influence drinking behavior in young adulthood, J Stud Alcohol Drugs, 71 (2010), 742-750.

[4]. M. P. Davoren, J. Demant, F. Shiely, and I. J. Perry, Alcohol consumption among university students in Ireland and the United Kingdom from 2002 to 2014: a systematic review, BMC Public Health, 16 (2016), 173-186.

[5]. H. W. Perkins, Surveying the damage: a review of research on consequences of alcohol misuse in college populations, J Stud Alcohol Supp, 14 (2002), 91-100.

[6]. E. L. Paul, B. McManus, and A. Hayes, "Hookups". Characteristics and correlates of college students' spontaneous and anonymous sexual experiences, J Sex Res, 37 (2000), 76-88.

[7]. K. M. Caldeira, A. M. Arria, K. E. O’Grady, E. M. Zarate, K. B. Vincent, and E. D. Wish, Prospective associations between alcohol and drug consumption and risky sex among female college students, J Alcohol Drug Educ, 53 (2009), 71-92.

[8]. J. A. Griffin, M. R. Umstattd, and S. L. Usdan, Alcohol use and high-risk sexual behavior among collegiate women: a review of research on alcohol myopia theory, J Am Coll Health, 58 (2010), 523-532.

[9]. M. E. Patrick and J. L. Maggs, Does drinking lead to sex? Daily alcohol-sex behaviors and expectancies among college students, Psychol Addict Behav, 23 (2009), 472-481.

[10]. A. N. Justus, P. R. Finn, and J. E. Steinmetz, The influence of traits of disinhibition on the association between alcohol use and risky sexual behavior, Alcohol Clin Exp Res, 24 (2000), 1028-1035.

[11]. R. Bø, J. Billieux, and N. I. Landrø, Which facets of impulsivity predict binge drinking?, Addict Behav Rep, 3 (2016), 43-47.

[12]. A. Birthrong and R. D. Latzman, Aspects of impulsivity are differentially associated with risky sexual behaviors, Pers Individ Dif, 57 (2014), 8-13.

[13]. H. De Wit, Impulsivity as a determinant and consequence of drug use: a review of underlying processes, Addict biol, 14 (2009), 22-31.

[14]. T. Duka, L. Trick, K. Nikolaou, M. A. Gray, M. J. Kempton, H. Williams, S. C. Williams, H. D. Critchley, and D. N. Stephens, Unique brain areas associated with abstinence control are damaged in multiply detoxified alcoholics, Biol Psychiatry, 70 (2011), 545-552.
[15]. T. Deckman and C. N. DeWall, Negative urgency and risky sexual behaviors: A clarification of the relationship between impulsivity and risky sexual behavior, Pers Individ Dif, 51 (2011), 674-678.

[16]. A. C. Black, T. J. McMahon, M. N. Potenza, L. E. Fiellin, and M. I. Rosen, Gender moderates the relationship between impulsivity and sexual risk-taking in a cocaine-using psychiatric outpatient population, Pers Individ Dif, 75 (2015), 190-194.

[17]. L. A. Nydegger, S. L. Ames, A. W. Stacy, and J. L. Grenard, Response inhibition moderates the association between drug use and risky sexual behavior, Subst Use Misuse, 49 (2014), 1457-1464.

[18]. R. M. Schuster, N. A. Crane, R. Mermelstein, and R. Gonzalez, The influence of inhibitory control and episodic memory on the risky sexual behavior of young adult cannabis users, J Int Neuropsycholl Soc, 18 (2012), 827-833.

[19]. D. L. Gullette and M. A. Lyons, Sexual sensation seeking, compulsivity, and HIV risk behaviors in college students, J Community Health Nurs, 22 (2005), 47-60.

[20]. L. Donohew, R. Zimmerman, P. S. Cupp, S. Novak, S. Colon, and $\mathrm{R}$. Abell, Sensation seeking, impulsive decision-making, and risky sex: Implications for risk-taking and design of interventions, Pers Individ Dif, 28 (2000), 1079-1091.

[21]. J. A. Turchik, J. P. Garske, D. R. Probst, and C. R. Irvin, Personality, sexuality, and substance use as predictors of sexual risk taking in college students, J Sex Res, 47 (2010), 411-419.

[22]. J. MacKillop, M. A. Celio, N. R. Mastroleo, C. W. Kahler, D. Operario, S. M Colby, et al., Behavioral economic decision making and alcohol-related sexual risk behavior, AIDS Behav, 19 (2015), 450-458.

[23]. J. M. Townshend, N. Kambouropoulos, A. Griffin, F. J. Hunt, and R. M. Milani, Binge drinking, reflection impulsivity, and unplanned sexual behaviour: impaired decision-making in young social drinkers, Alcohol Clin Exp Res, 38 (2014), 1143-1150.

[24]. S. R. Carlson, S. C. Johnson, and P. C. Jacobs, Disinhibited characteristics and binge drinking among university student drinkers, Addict Behav, 35, (2010), 242-251.

[25]. A. Mehrabian and J. A. Russell, A questionnaire measure of habitual alcohol use, Psychol Rep, 43 (1978), 803-806.

[26]. J. M. Townshend and T. Duka, Patterns of alcohol drinking in a population of young social drinkers: a comparison of questionnaire and diary measures, Alcohol Alcohol, 37 (2002), 187-192.

[27]. J. H. Patton, M. S. Stanford, and E. S. Barratt, Factor structure of the Barratt Impulsiveness Scale, J Clin Psychol, 51 (1995), $768-774$.

[28]. R. Torrubia, C. Avila, J. Moltó, and X. Caseras, The Sensitivity to Punishment and Sensitivity to Reward Questionnaire (SPSRQ) as a measure of Gray's anxiety and impulsivity dimensions, Pers Individ Dif, 31 (2001), 837-862.

[29]. A. J. Caswell, M. A. Celio, M. J. Morgan, T. Duka, Impulsivity as a multifaceted construct related to excessive drinking among $U K$ students, Alcohol Alcohol, 51 (2015), 77-83.

[30]. N. Kambouropoulos and P. K. Staiger, Personality, behavioural and affective characteristics of hazardous drinkers, Pers Individ Dif, 42 (2007), 213-224. 
[31]. S. Dawe and N. J. Loxton, The role of impulsivity in the development of substance use and eating disorders, Neurosci Biobehav Rev, 28 (2004), 343-351.

[32]. L. Clark, J. P. Roiser, T. W. Robbins, and B. J. Sahakian, Disrupted 'reflection' impulsivity in cannabis users but not current or former ecstasy users, J Psychopharmacol, 23 (2009), 14-22.

[33]. A. J. Lawrence, J. Luty, N. A. Bogdan, B. J. Sahakian, and L. Clark, Problem gamblers share deficits in impulsive decisionmaking with alcohol-dependent individuals, Addiction, 104 (2009), 1006-1015.

[34]. P. Banca, I. Lange, Y. Worbe, N. A. Howell, M. Irvine, N. A.
Harrison, M. Moutoussis, and V. Voon, Reflection impulsivity in binge drinking: behavioural and volumetric correlates, Addict Biol, 21 (2016), 504-515.

[35]. R. Bø, M. Aker, J. Billieux, and N. I. Landrø, Binge drinkers are fast, able to stop-but they fail to adjust, J Int Neuropsychol Soc, 22 (2016), 38-46.

[36]. C. Nederkoorn, M. Baltus, R. Guerrieri, and R. W. Wiers, Heavy drinking is associated with deficient response inhibition in women but not in men, Pharmacol Biochem Behav, 93 (2009), 331-336.

[37]. M. L. Cooper, P. K. Wood, H. K. Orcutt, and A. Albino, Personality and the predisposition to engage in risky or problem behaviors during adolescence, J Pers Soc Psychol, 84 (2003), 390-410. 